Ann. Génét. Sél. anim., 1976, 8 (3), 315-329.

\title{
ESTIMATION \\ DE QUELQUES PARAMÈTRES DÉMOGRAPHIQUES DU PUR SANG ANGLAIS EN FRANCE
}

\author{
B. LANGLOIS \\ avec la collaboration technique d'Isabelle MAHEUT \\ Station de génétique quantitative et appliquée, \\ Centre national de Recherches zootechniques, I. N.R. A., \\ 78350 Jouy en Josas
}

\section{RÉSUMÉ}

L'étude fondée sur les données du Stud-Book français a permis d'estimer l'intervalle de génération chez le Pur Sang anglais à 10,5 ans. Cette valeur élevée est due à un âge moyen de mise à la reproduction de 6,9 ans chez les mâles $(n=23$ I) et de 4,9 ans chez les femelles $(n=930)$ ainsi qu'à une réforme tardive à 16,3 ans en moyenne chez les mâles $(n=477)$ et 14,3 ans chez les femelles $(n=930)$.

Par ailleurs un étalon a en moyenne 7,03 produits sevrés par an, ce qui correspond à un taux de fertilité annuel de $0,5^{8}$ pour les juments. Sur l'ensemble de sa carrière, il laisse en moyenne 52,4 produits, une poulinière 5,3 .

Près de 15 p. roo des mâles n'ayant leur première saillie qu'à l'âge de 9 ans et plus et environ $30 \mathrm{p}$. Ioo d'entre eux ne laissant que $2,5 \mathrm{p}$. Ioo des produits, il semble possible par une conduite plus systématique de l'élevage de réduire dans des proportions appréciables l'intervalle de génération par la voie paternelle.

Pour les femelles le taux théorique de renouvellement annuel a été estimé de l'ordre de 5 p. roo. Il semble donc également possible en réformant plus activement les femelles peu fertiles ou âgées d'intervenir par cette voie sur l'intervalle de génération.

Enfin, les effectifs moyens disponibles pour le contrôle de la descendance, faibles pour les étalons, sont négligeables pour les juments.

Il apparaît en conclusion que la structure de reproduction de la population française de Pur Sang anglais semble pouvoir être améliorée. Sans modification de la structure de sélection cela permettrait déjà d'obtenir un progrès génétique plus rapide.

\section{INTRODUCTION}

L'étude démographique de la population française de Pur Sang anglais s'impose. En effet, bien que les éleveurs et professionnels s'en fassent une idée intuitive, aucun chiffre précis n'est disponible à ce sujet. Pour l'intervalle de génération par exemple, 
nous n'avons trouvé qu'une seule référence bibliographique (STEELE, I944) qui l'estime à I2,I ans. En ce qui concerne la carrière reproductive des étalons et poulinières, les renseignements précis sont également rares et concernent rarement le Pur Sang. Compte tenu de l'importance de ces connaissances dans la conception des programmes d'amélioration génétique, nous nous proposons d'en fournir ici quelques estimations à partir des données du Stıd-Bookfrançais, registre des chevaux de Pur Sang nés ou importés en France. Les paramètres étudiés qui concernent aussi bien les reproducteurs des deux sexes sont l'intervalle de génération, 1'âge moyen, l'âge à la première saillie, 1'âge à la mort ou à la réforme, la durée de vie reproductive, le nombre annuel et le nombre total de descendants. Cette étude s'inscrit, pour le cheval, dans un thème initié dans cette même revue pour la race ovine Sarde (LAUVERGNE et al., 1973). L'approche des problèmes de démographie génétique y est traitée avec beaucoup de similitude.

\section{MATÉRIEL ET MÉTHODES}

Ce paragraphe se trouve réparti pour tous les paramètres étudiés au niveau des résultats où il s'intègre plus aisément en raison des particularités propres à chaque estimation. Précisons toutefois que la méthode dite " des cohortes ", habituelle à la démographie, a été employée comme nous le verrons dans la suite pour définir les échantillons de travail. Précisons également qu'une optique strictement descriptive nous a fait écarter toute analyse statistique fine des résultats. La présentation par variable des distributions de fréquences, des moyennes et des modes, nous a en effet paru suffisante pour que les conséquences pour la sélection puissent en être aussitôt déduites.

\section{RÉSULTATS ET DISCUSSION}

\section{I. - Estimation de l'intervalle de génération}

L'intervalle de génération se définit comme étant l'âge moyen des reproducteurs à la naissance de leurs descendants destinés à la reproduction. En pratique, lorsque la durée d'utilisation des mâles et des femelles est différente les quatre composantes suivantes doivent être précisées :

$\mathbf{I}^{\mathbf{0}}$ âge des femelles de la génération $(n-\mathrm{I})$ à la naissance de leurs fils reproducteurs de la génération $n$;

$2^{\circ}$ âge des mâles de la génération $(n-\mathrm{I})$ à la naissance de leurs fils reproducteurs de la génération $n$;

$3^{\circ}$ âge des femelles de la génération ( $n$-I) à la naissance de leurs filles reproductrices de la génération $n$;

$4^{0}$ âge des mâles de la génération $(n-r)$ à la naissance de leurs filles reproductrices de la génération $n$.

Les deux premières composantes ont donc été estimées par les différences de l'année de naissance des 438 étalons ayant fait la monte en r 973 avec celles de leurs parents, les deux suivantes en calculant par la même méthode ces différences pour un échantillonnage de 556 poulinières en service en r973. L'âge moyen de ces reproducteurs a, par ailleurs, été calculé de façon à fournir d'autres estimations de l'intervalle de génération. Les résultats détaillés de ces calculs sont rapportés figure I sous forme 
de pyramides des âges pour permettre une vision plus globale. Il en résulte que l'âge moyen des reproducteurs actuels à la naissance de leurs descendants, II, 4 ans, est supérieur à l'intervalle de génération qui est estimé à Io,5 ans. Deux phénomènes peuvent être à l'origine de ce fait : tout d'abord un choix préférentiel des reproducteurs de la génération à venir dans les premières années de production de la génération en activité. Ceci serait surtout marqué pour les étalons où l'on note (côté droit du graphique) de notables variations dans les histogrammes. En second lieu, un vieillissement de la population française actuelle marqué surtout du côté des mâles. les distributions côté femelle (à gauche sur le graphique) demeurant sensiblement équivalentes, pourrait également intervenir.

(A) AGE DES REPRODUCTEURS : MOY. 11,4 ans.

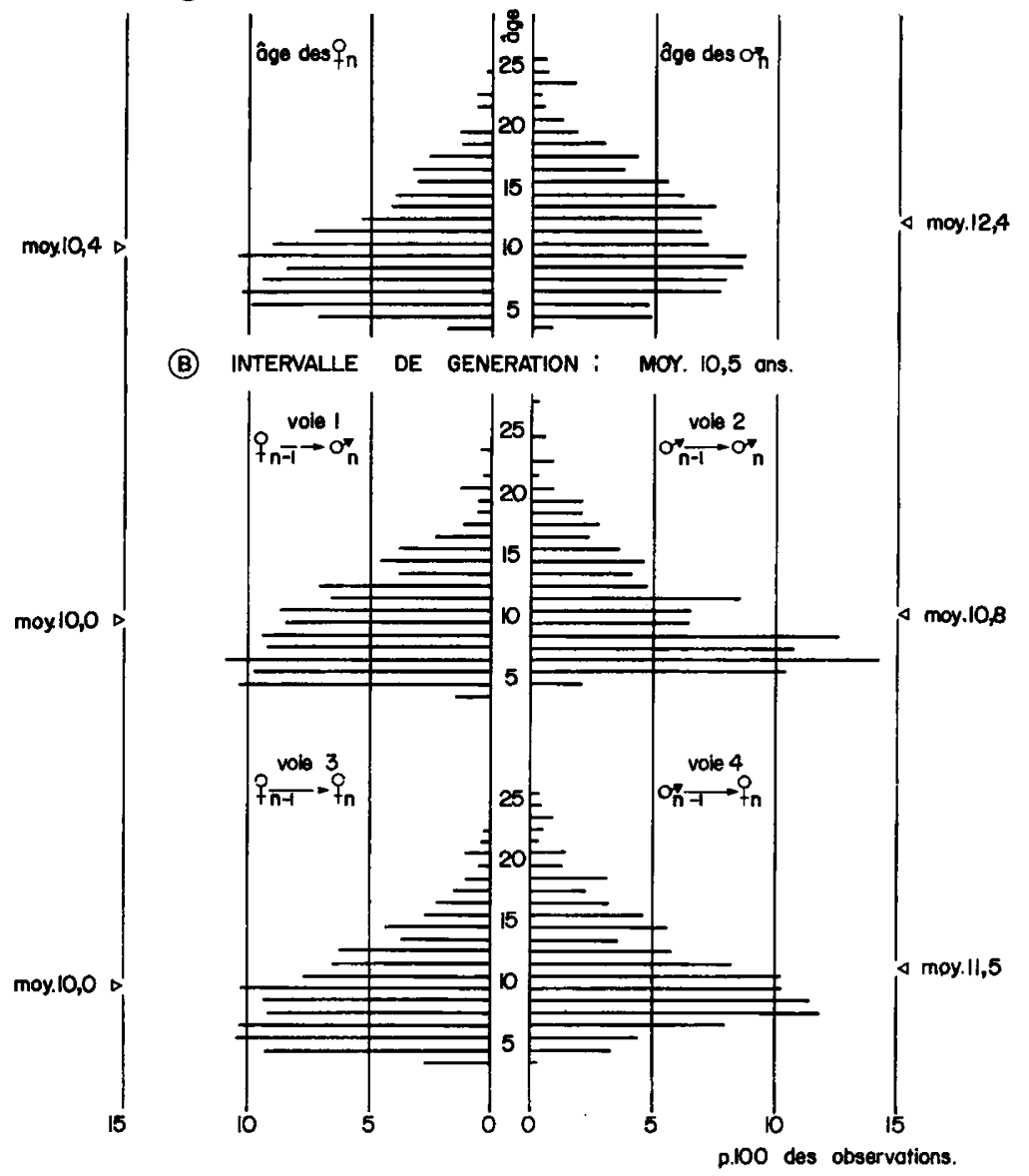

FIG. I. - Estimation de l'intervalle de generation Estimation of the generation interval

A Age of breeding animals : Mean II.4 years

B Generation interval : Mean 10.5 years

I1 ne nous est pas possible ici compte tenu de nos données de trancher entre ces deux hypothèses. Remarquons cependant que l'intervalle de génération déjà très 
grand est paradoxalement plus long par la voie mâle (II ans) qu'il ne l'est par la voie femelle (Io ans) où les possibilités théoriques de renouvellement sont pourtant beaucoup moins importantes.

\section{II. - Composantes de la carrière reproductive des étalons}

Les composantes de la carrière reproductive des étalons sont l'âge à la première saillie, l'âge à la réforme ou à la mort, la durée de vie reproductive, le nombre moyen de produits sevrés par an, son évolution avec l'âge et le nombre total de ses descendants. L'analyse descriptive de la carrière des étalons morts ou réformés depuis 1945 a donc été abordée. Seule la production en race pure a été considérée. Les effectifs sont parfois faibles en raison d'un taux de réforme annuel bas. Mais pour avoir un échantillon plus large il nous aurait fallu remonter beaucoup plus loin dans le temps et notre échantillon n'aurait plus été représentatif de la population actuelle. Notons encore que certaines difficultés dans la collecte des données nous ont empêchés de reconstituer totalement toutes les carrières étudiées. C'est pourquoi les effectifs d'estimation des différents paramètres pourront varier.

\section{Age à la première saillie.}

Physiologiquement selon Nishikawa et HaFez (Ig62) les testicules des mâles, parfois en place à la naissance, descendent le plus souvent vers l'âge de 2 à 3 semaines. Leur croissance rapide ne débute cependant que vers le $I^{\mathrm{e}} \mathrm{e}$ mois en commençant par le gauche. Le premier sperme est produit vers I an mais l'on considère généralement que le jeune cheval n'est apte à la saillie fécondante qu'à l'âge de 2 ans. La mise à profit de cette possibilité n'est pas toujours compatible avec le service demandé aux animaux. Elle supprime en effet toute possibilité de sélection sur les performances individuelles et il est exclu pour les chevaux de course que cette méthode soit intéressante.

A

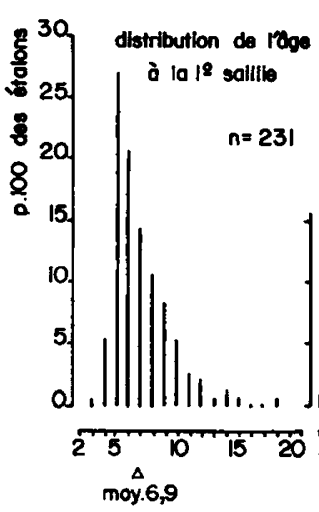

B

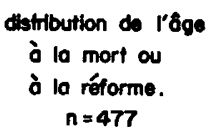

distribution de l'óge

la réforme.

$n=477$

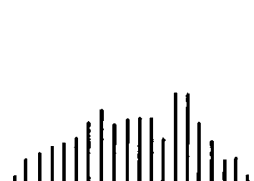

c

distribution de la durte

de vie reproductive.

$n=259$

FIG. 2. - Composantes de la carrière reproductive des étalons Components of the reproductive life of stallions
A Distribution of age at first service
B Distribution of age at death or culling
C Distribution of length of reproductive life 
Notre étude portant sur 23I mâles, donne un âge moyen à la première saillie de 6,9 ans. Sa distribution (fig. 2) très dissymétrique montre cependant un mode bien prononcé à 5 ans. Ceci laisse supposer que la majorité des étalons entrent au haras après une carrière en course à 2,3 et 4 ans. En revanche, un pourcentage non négligeable (de l'ordre de $I_{5} \mathrm{p}$. Ioo) ne fait sa première saillie qu'à 9 ans et plus. Ce sont pour la plupart des chevaux qui, ayant gagné régulièrement dans les courses plates de niveau moyen ou dans les courses à obstacles, se trouvent mis à la reproduction en raison de leur résistance ou de leur longévité sportive. Il peut également s'agir d'importations, mais il faut remarquer que leur arrivée à l'élevage augmente considérablement (près de 2 ans) l'âge moyen à la première saillie des étalons qui pourrait se situer à 5 ans. Dans une optique de sélection'sur les résultats des courses plates, elle ne nous paraît donc pas souhaitable car elle allonge l'intervalle de génération au profit d'une sélection très discutable.

\section{Age à la réforme ou à la mort.}

L'âge à la réforme ou à la mort des étalons peut être très différent selon le contexte de l'élevage et selon les individus considérés. SAsmowskI (I96r), en Pologne, l'estime à 6,7 ans en moyenne pour 935 étalons privés et à I3,I ans pour 132 étalons de Pur Sang de 1'État. Il précise que 24 p. 100 des mâles utilisés en monte publique sont réformés après une saison de monte, à 4 ou 5 ans. Il est évident que nous sommes là assez loin des conditions françaises comme nous allons maintenant le préciser.

Dans l'estimation de l'âge moyen à la réforme ou à la mort il était possible de distinguer ces deux éventualités. En fait, beaucoup d'étalons étant signalés morts lorsque leur réforme est suivie d'abattage cela peut paraître discutable. La distribution bimodale de ce paramètre (fig. 2) dont la moyenne se situe à 16,3 ans nous laisse, cependant supposer la superposition de deux phénomènes : mort naturelle d'une part, réforme volontaire d'autre part. En fait, l'analyse plus fine et séparée des 253 étalons déclarés morts et des 224 réformés de notre effectif nous permet, en tenant compte du phénomène de chevauchement précité, d'avancer les propositions suivantes :

Cinquante pour cent au moins des étalons sont réformés avant leur mort. Ces réformes interviennent en deux étapes : une première vague centrée sur l'âge de I2 ans et répartie entre 9 et $I_{5}$ ans correspond vraisemblablement à une sélection sur descendance. Une seconde beaucoup plus resserrée, autour de I9 ans, intervient juste avant 1'âge normal de la mort et correspond probablement à l'élimination des étalons devenus trop vieux pour assurer convenablement leur fonction de reproducteur. Enfin, la mort par accident ou maladie peut intervenir à tout âge mais voit son importance culminer entre 20 et $2 I$ ans, âges qui peuvent être considérés comme l'espérance de vie normale d'un étalon de Pur Sang en France.

\section{Durée de vie reproductive.}

Manunta (I953) étudiant un effectif de I 569 chevaux Sardes morts de 1948 à I952 estime la durée de vie reproductive des mâles de cette race à II,8 ans. Dans notre cas, en faisant pour chaque étalon la différence entre l'âge à la réforme ou à la mort et l'âge à la première saillie nous avons également estimé ce paramètre sur un effectif de 259. La moyenne générale se situe à 9,7 ans et il n'apparaît qu'une très 
faible différence entre les morts et les réformés. Ces résultats sont comparables à ceıx que l'on aurait obtenus en faisant directement la différence des moyennes des âges de réforme ou de mort avec la moyenne de l'âge à la première saillie. On peut cependant noter la grande étendue de la distribution (fig. 2) elle-même déterminée par la grande variation de 1'âge à la réforme ou à la mort.

\section{Nombre de descendants d'un étalon.}

Le nombre de descendants laissés par un étalon au cours de sa carrière est déterminé par le nombre moyen de juments qu'il saillit annuellement, par la durée de sa vie reproductive et par son taux moyen de fertilité. Il nous a donc paru opportun d'examiner l'évolution en fonction de l'âge du nombre moyen de produits sevrés annuellement par étalon. A cet effet, 2707 années de monte, correspondant à un total de 19022 produits ont été analysées. Il en résulte une moyenne de 7,03 produits par an et par mâle, le maximum dénombré pour une année ayant été de 3o. L'évolution en fonction de 1'âge de cet effectif moyen est reportée figure 3 . Il y apparaît que le nombre annuel des descendants d'un étalon diminue avec l'âge, sa production la plus active se situant approximativement de 5 à $\mathbf{I}_{5}$ ans.
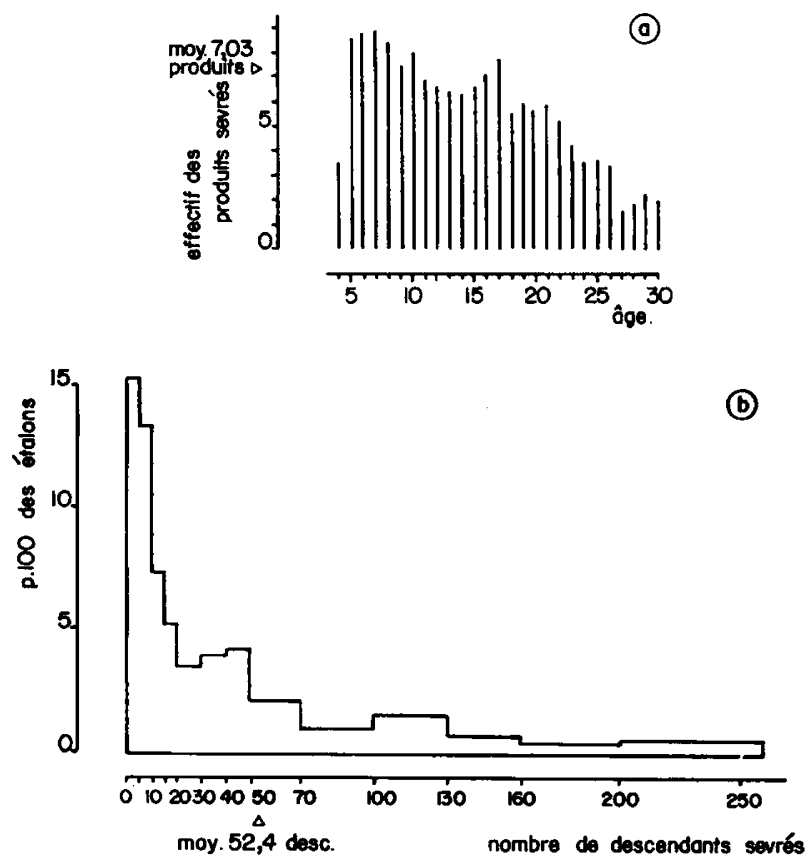

Fig. 3. - Nombre de descendants sevrés par étalon

Number of offsprings weaned per stallion

a par an en fonction de l'âge per year according to age

b au total en fonction des étalons total according to stallions

Par ailleurs la production complète de I5I étalons durant toute leur carrière a pu être reconstituée. Cela correspondait à un effectif de 7954 chevaux soit une moyenne de $52,4 \mathrm{I}$ produits par père, avec un maximum de 253 pour l'un d'entre eux. 
La distribution de l'effectif des étalons en fonction de l'importance de leur descendance a pu ainsi être établie (fig. 3). Il en découle, étant donné la très grande variation de la taille de la famille, que l'effectif génétique efficace des étalons de Pur Sang est très inférieur à leur nombre réel. En effet on peut noter qu'approximativement $30 \mathrm{p}$. roo des mâles, les moins productifs, ne laissent que 2,5 p. Ioo des produits. A l'inverse seulement $20 \mathrm{p}$. Inn d'entre eux fournissent $60 \mathrm{p}$. roo de l'effectif de la génération suivante.

\section{L'étalon type.}

Il nous est maintenant permis en récapitulant les différents résultats précédents de dresser rapidement le profil type de l'étalon de Pur Sang en France. Ce dernier a environ $\mathrm{I} 2$ ans et est entré au haras vers 6 ou 7 ans après avoir été exploité en course. Son espérance de vie est de $20-2 I$ ans mais il sera réformé vers 16 ans après 9 à ro ans de service. La moyenne annuelle de sa production sera de 1'ordre de 7 descendants. A la fin de sa carrière une cinquantaine de produits pourront être dénombrés. A ce portrait il serait bien entendu préférable de substituer le suivant : âge, Io ans, entré au haras à 5 ans, réformé à 15 ans après une production annuelle de l'ordre de 15 à 20 descendants, soit au total en fin de carrière 150 à 200 produits.

Remarquons toutefois que 1'utilisation, en France, d'un grand nombre d'étalons de Pur Sang pour le croisement, fausse dans de notables proportions les données du problème. Il serait sans doute souhaitable de les distinguer clairement de ceux qui contribuent réellement à l'amélioration de la race de course qu'est le Pur Sang anglais.

\section{III. - Composantes de la carrière reproductive des poulinières}

L'étude des composantes de la carrière reproductive des femelles soit 1'âge à la première saillie, l'âge à la réforme ou à la mort, la durée de vie reproductive, la fertilité annuelle et le nombre total de descendants, a été conduite sur un effectif de 930 poulinières de Pur Sang anglais mortes ou réformées de 1965 à 1970 en France. Par ailleurs les renseignements concernant les viduités, les avortements, la mortalité au lait et les gestations gémellaires étant disponibles nous avons pu estimer certains paramètres de l'aptitude maternelle de ces juments. Nous avons également tenu compte des produits obtenus en croisement mais leur proportion étant faible (I,25 p. Ioo de la production) nous n'avons pas jugé intéressant de les étudier à part. Voyons maintenant les résultats de cette analyse.

\section{Age à la première saillie.}

D'une manière générale 1'âge à la puberté semble sujet à des fluctuations assez importantes en fonction des conditions d'alimentation et de facteurs génétiques comme cela a été mis en évidence chez d'autres espèces (LEGAULT, I973 chez les porcs ; WIITBANK et al., I 966 chez les bovins). Chez les juments cette maturité n'apparaît le plus fréquemment que vers l'âge de 15 à 18 mois (NiSHIKAWA et HAFEZ, I962). Cependant, en raison du saisonnement sexuel elle semble très influencée par le mois de naissance et souvent elle ne survient que vers 25 à 28 mois. Physiologiquement les juments ont donc une première saillie envisageable à l'âge de 2 ans. Cette possibilité n'est cependant que rarement mise à profit et les quelques données de la littéra- 
ture (Detkens et Konopinski, I954; Matassino, I962 ; Salerno et MonteMURRO, I965 ; BELIIITI et PAGANo, I967 ; de FrANCISCI et al., I972) nous permettent de situer approximativement vers 4 ou 5 ans l'âge à la première saillie. Mais ces données hétérogènes ne concernent pas le Pur Sang anglais aussi voyons ce que donne l'examen des 930 juments de notre échantillon. Comme le montre la figure 4 le mode de la distribution est à 4 ans et laisse supposer une sélection d'après les performances en course à 2 et 3 ans. La moyenne se situe à 4,9 ans illustrant le fait que l'entrée au haras des juments est beaucoup mieux synchronisée que ne l'est celle des étalons. Cette tendance semble malheureusement se relâcher quelque peu ces derniers temps. En effet, un sondage sur 264 poulinières ayant mis bas pour la première fois en 1973 ne permet d'estimer l'âge à la première saillie qu'à 5,4 ans en moyenne.

A

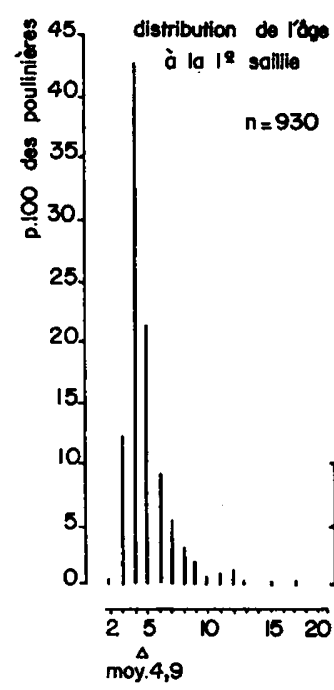

B

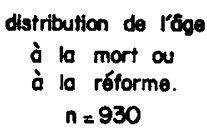

C

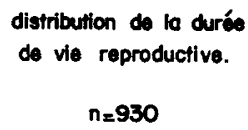

distribution de la durée

$\mathrm{n}=930$

FIG. 4. - Composantes de la carrière reproductive des poulinières Components of reproductive life of mares
A Distribution of age at first mating
B Distribution of age at death or culling
C Distribution of length of reproductive life

\section{Age à la mort ou à la réforme.}

De même que pour les étalons nous avons pu distinguer les morts des réformes, conscients du fait que certaines juments réformées puis abattues sont déclarées mortes. Ceci nous a permis en considérant séparément ces deux catégories de mettre en évidence sur 365 juments, trois périodes successives de réformes se situant respectivement autour des âges de II, I5 et 20 ans dont la plus intense paraît être la seconde. Sur les 565 poulinières restantes (déclarées " mortes ") nous avons, compte tenu du résultat précédent, estimé l'espérance de vie des poulinières de 1'ordre de $I 7$ à $I 8$ ans. Cela est sensiblement plus faible que pour les étalons mais nous ne fournissons ce résultat approximatif qu'avec réserve. Il coïncide néanmoins avec les résultats trouvés par Comfort (1956, I959 et 1962) sur les données du General Stud Book anglais. 
Au total $(n=930)$ nous obtenons un âge à la réforme ou à la mort dont la distribution étalée (fig. 4) a un mode à $I_{5}$ ans et une moyenne à $1_{4,3}$ ans. Nous pouvons en conclure que les poulinières de Pur Sang anglais sont dans l'ensemble utilisées à la mesure de leurs capacités de production. Les réformes n'interviennent sans doute que dans le cas de difficultés de reproduction. Leur exploitation plus intensive que celle des étalons les conduit, dans l'ensemble, à une durée de vie plus faible.

\section{Durée de vie reproductive.}

La durée de vie reproductive que Manunta (I953) estime à 13 ans chez les chevaux Sardes et que De CARvahLo et al. (I972) dans la race brésilienne Mangalarga Paulista évalue à 5,4 ans, se calcule en soustrayant 1'âge à la première saillie de l'âge à la réforme ou à la mort. La distribution de cette variable est reportéc figure 4. Les modes à 5 et II ans de production illustrent les taux de réformes réalisés chez les juments autour de II et I5 ans. La moyenne se situe à 9,6 ans, la dispersion est importante, comme celle de l'âge à la réforme ou à la mort.

\section{Nombre de descendants d'une poulinière.}

Le nombre de descendants laissés par une poulinière dépend de sa fertilité et de la durée de sa vie reproductive. Enn considérant l'ensemble de la carrière des 930 juments étudiées soit un total de 8896 années de production, nous avons pu dresser le tableau I qui nous donne le résultat moyen pour une jument dans sa vie. Au bas du tableau, les paramètres usuels tels que taux de fertilité, de mortalité et de prolificité, ont pu en outre être calculés. Le nombre de produits sevrés par une poulinière soit 5,3 en moyenne nous semble le critère le plus synthétique de sa capacité de production. Sa distribution (fig. 5) est, comme l'exprime le mode à 4 descendants, légèrement dissymétrique en faveur des familles de petite taille. Cela rend illusoire, pensons nous, la pratique d'un contrôle de descendance chez les poulinières. Nous pouvons par ailleurs, compte tenu de ces résultats, évaluer à 2,65 pour un sex-ratio de I le nombre de femelles laissées par une jument pour assurer son remplacement. Cela laisse donc une possibilité de sélection non négligeable que l'on peut situer de l'ordre de 50 p. Ioo des juments. En effet, si nous considérons que pour des raisons diverses (accidents, maladies, malformations...) deux seulement de ces pouliches arrivent à l'âge de mise à la reproduction, le taux de remplacement des juments est de $\mathrm{I} / 2$. Chaque jument produisant en moyenne 9,6 ans, nous pouvons également estimer dans l'hypothèse de stabilité de la population, que le taux théorique de renouvellement annuel est de $I / 2: 9,6=0,05$, ce qui est très faible.

Le taux moyen de sevrage bas $(57,8$ p. I00) dû essentiellement à une fertilité de départ très moyenne (67,3 p. roo) explique en partie cette faible rotation du cheptel femelle. En effet, devant cette situation, les éleveurs tenus par les impératifs de l'amortissement des poulinières qui doit être prévu sur 4 à 5 produits, sont conduits à maintenir leurs femelles le plus longtemps possible à l'élevage. Or il est possible qu'une telle attitude conduise à un enchaînement de faits aggravant encore ces résultats. En effet, Wussow et HARTwIG (I956), BAIN (I969) ont montré que la fertilité décroîssait avec l'âge, ce que nos résultats (fig. 5) confirment. Par ailleurs nous avons pu montrer qu'à âge égal une première saillie paraît sensiblement plus fertile qu'en moyenne. En effet, la comparaison des résultats de la première saillie aux résultats 


\section{TABLEAU I}

Performances de reproduction de la poulinière de Pur Sang type Mean reproductive performances of the Thoroughbred mare

\begin{tabular}{|c|c|c|}
\hline & $\begin{array}{l}\text { Total pour } 930 \text { 우우 } \\
\text { (Total for } 930 \text { 우우) }\end{array}$ & $\begin{array}{l}\text { Moyenne pour } 1 \text { ㅇ } \\
\text { (Mean for } 1 \text { i) }\end{array}$ \\
\hline $\begin{array}{l}\text { 1. Nombre d'années étudiées } \ldots \ldots \ldots \ldots \ldots \ldots \ldots \ldots \ldots \\
\text { (Number of years studied) }\end{array}$ & 8896 & 9,55 \\
\hline $\begin{array}{l}\text { 2. Nombre d'années " sans renseignements " } . . . \ldots \ldots \ldots \\
\text { (Number of years " without information })\end{array}$ & 114 & 0,12 \\
\hline $\begin{array}{l}\text { 3. Nombre d'années " non saillie } \$ . . . \ldots \ldots \ldots \ldots \ldots \\
\text { (Number of years a without mating })\end{array}$ & 247 & 0,27 \\
\hline 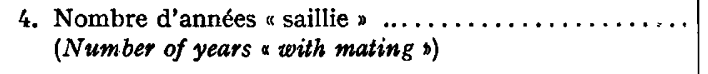 & 8535 & 9,18 \\
\hline $\begin{array}{l}\text { 5. Nombre de viduités } \ldots \ldots \ldots \ldots \ldots \ldots \ldots \ldots \ldots \ldots \ldots \\
\text { (Number of years without gestations) }\end{array}$ & 2790 & 3,00 \\
\hline $\begin{array}{l}\text { 6. Nombre de fécondations } \ldots \ldots \ldots \ldots \ldots \ldots \ldots \ldots \ldots \ldots \\
\text { (Number of fertilizations) }\end{array}$ & 5745 & 6,18 \\
\hline $\begin{array}{l}\text { 7. Nombre d'avortements } \ldots \ldots \ldots \ldots \ldots \ldots \ldots \ldots \ldots \ldots \\
\text { (Number of abortions) }\end{array}$ & 357 & 0,38 \\
\hline $\begin{array}{l}\text { 8. Nombre de mises bas } \ldots \ldots \ldots \ldots \ldots \ldots \ldots \ldots \ldots \ldots \\
\text { (Number of foalings) }\end{array}$ & 5388 & 5,79 \\
\hline $\begin{array}{l}\text { 9. Nombre de gestations gémellaires } \ldots \ldots \ldots \ldots \ldots \ldots \ldots \\
\text { (Number of twin-pregnancies) }\end{array}$ & 93 & 0,10 \\
\hline $\begin{array}{l}\text { 10. Nombre de naissances gémellaires } \ldots \ldots \ldots \ldots \ldots \ldots \\
\text { (Number of twin-foalings) }\end{array}$ & 40 & 0,04 \\
\hline $\begin{array}{l}\text { 11. Nombre de poulains nés } \ldots \ldots \ldots \ldots \ldots \ldots \ldots \ldots \ldots \ldots \\
\text { (Number of foals born) }\end{array}$ & 5428 & 5,83 \\
\hline $\begin{array}{l}\text { 12. Nombre de morts-nés } \ldots \ldots \ldots \ldots \ldots \ldots \ldots \ldots \ldots \ldots \\
\text { (Number of stillborn foals) }\end{array}$ & 222 & 0,24 \\
\hline $\begin{array}{l}\text { 13. Nombre de morts au lait } \ldots \ldots \ldots \ldots \ldots \ldots \ldots \ldots \ldots \\
\text { (Number of foals dead at suckling) }\end{array}$ & 269 & 0,29 \\
\hline \multirow[t]{2}{*}{$\begin{array}{l}\text { 14. Nombre de poulains sevrés } \ldots \ldots \ldots \ldots \ldots \ldots \ldots \ldots \ldots \\
\text { (Number of weaned foals) }\end{array}$} & 4936 & 5,31 \\
\hline & & $(\%)$ \\
\hline $\begin{array}{l}\text { Taux de fertilité } \ldots \ldots \cdots \cdots \cdots \cdots \cdots \cdots \cdots \cdots \cdots \cdots \cdots \cdots \\
(\text { Fertility rate })\end{array}$ & $6 / 4$ & 67,3 \\
\hline $\begin{array}{l}\text { Taux de fertilité apparente } \ldots \ldots \ldots \ldots \ldots \ldots \ldots \ldots \ldots \ldots \\
\text { (Apparent fertility rate) }\end{array}$ & $8 / 4$ & 63,1 \\
\hline $\begin{array}{l}\text { Taux de productivité numérique } \ldots \ldots \ldots \ldots \ldots \ldots \cdots \cdots \\
\text { (Numerical productivity rate) }\end{array}$ & $14 / 4$ & 57,8 \\
\hline $\begin{array}{l}\text { Taux d'avortements } \ldots \ldots \ldots \cdots \cdots \cdots \cdots \cdots \cdots \cdots \cdots \cdots \\
\text { (Abortion rate) }\end{array}$ & $7 / 6$ & 6,2 \\
\hline $\begin{array}{l}\text { Taux de mortinatalité } \ldots \ldots \ldots \ldots \ldots \ldots \ldots \ldots \cdots \cdots \\
\text { (Stillborn rate) }\end{array}$ & $12 / 11$ & 4,1 \\
\hline $\begin{array}{l}\text { Taux de mortalité au lait. } \ldots \ldots \ldots \ldots \ldots \ldots \ldots \ldots \ldots \cdots \cdots \\
\text { (Preweaning mortality rate) }\end{array}$ & $13 / 11-12$ & 5,2 \\
\hline $\begin{array}{l}\text { Taux de prolificité. } \ldots \ldots \cdots \cdots \cdots \cdots \cdots \cdots \cdots \cdots \cdots \cdots \\
\text { (Prolificacy) }\end{array}$ & $11 / 8$ & 100,7 \\
\hline
\end{tabular}


d'ensemble en fonction de l'âge leur est nettement favorable. Ceci peut s'expliquer par le fait que les poulinières considérées ne sont pas fatiguées par des gestations antérieures et que n'étant pas allaitantes l'effet dépressif de la lactation sur la fertilité, général à toutes les espèces domestiques, ne se manifeste pas. Il peut cependant également s'agir du fait que de nombreuses juments sortant de l'entraînement et essayées à la reproduction ne sont pas conservées ni même inscrites au Stud-Book lorsqu'elles ne remplissent pas. Cela corroborerait ce que pensent les éleveurs qui déclarent avoir beaucoup plus de difficultés avec les primipares. Nous sommes cependant fondés d'après ces résultats à nous demander si le maintien à l'élevage de poulinières âgées, en augmentant leur proportion et en conduisant à un taux de renouvellement annuel très faible $(5 \mathrm{p}$. Ioo) diminuant ainsi le pourcentage de primipares, n'agit pas défavorablement sur la fertilité moyenne de la population. Il nous semble finalement, les reproductrices étant au maximum de leurs facultés entre 5 et Io ans, qu'il y aurait avantage à réformer très activement les femelles de plus de Io ans.

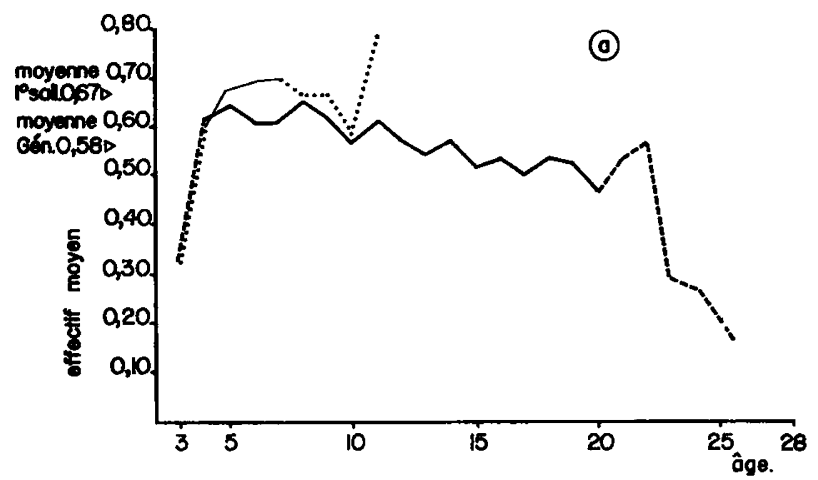

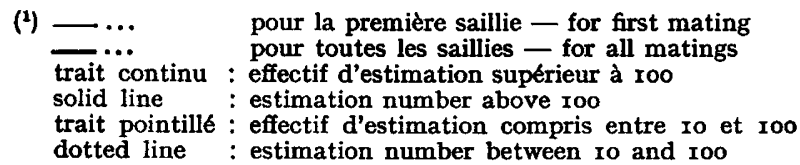

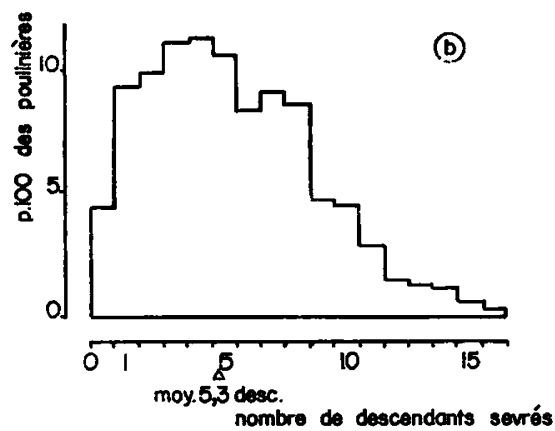

Fig. 5. - Nombre de descendants sevrés par poulinière

Number of offsprings reaned per mare

a par an en fonction de l'age ( ${ }^{(1)}$

per year according to age (1)

b au total en fonction des poulinières total according to mares 
Enfin le tableau I nous permet de faire le point sur les gestations gémellaires qui représentent en France pour le Pur Sang anglais I,09 p. roo des saillies et I,62 p. 100 des gestations. Cette dernière proportion varie selon les auteurs de $2 \mathrm{p}$. IOo (ZwoLINSKr, I966) à 3,2 p. IO0 (KoRoL,Kov, I95I). Ces études montrent que très peu d'entre elles aboutissent à un résultat intéressant. Les ovulations multiples seraient en revanche assez fréquentes, pouvant atteindre selon ARTHUR (I958) jusqu'à 35 p. Ioo des ovulations, mais elles aboutiraient le plus souvent à des mortalités embryonnaires ou à l'avortement précoce. Dans notre étude nous avons relevé 93 gestations doubles dont 53 se sont traduites par des avortements. Il ne semble pas qu'il $y$ ait un effet de l'âge ni que le phénomène soit spécifique de quelques juments. Sur 40 gestations gémellaires arrivées à terme 27 ont donné 2 morts-nés, 6, 2 morts au lait, 6 encore, I mort-né et I sevré, I enfin, I mort au lait et I sevré, aucune n'a conduit au sevrage des deux poulains. Le taux de prolificité $(I, 007)$ peut donc être en pratique évalué à I sans risque d'erreur.

\section{La jument type.}

Il nous est possible maintenant comme nous l'avons fait pour les étalons de brosser rapidement le portrait de la poulinière de Pur Sang en France. Son âge est l'environ Io ans, elle est entrée au haras vers 4 ou 5 ans après une carrière en course. Son espérance de vie est de l'ordre de $\mathrm{I} 7$ à $\mathrm{I} 8$ ans mais elle sera réformée un peu avant vers I4 ou I5 ans, ses possibilités de production étant épuisées. Sa fertilité annuelle faible $(0,67)$ ne la conduira à sevrer que $0,5^{8}$ produit par an soit I tous les r,7 ans. En fin de carrière, après 9 à ro années de production, le nombre de ses descendants sera de l'ordre de 5 . Par ailleurs, on peut noter que durant sa vie reproductive environ une sur ro connaîtra une gestation gémellaire, une sur 8 sera mise au repos une année, une sur 4 aura un poulain mort-né, une sur 3 ou 4 aura un poulain mort au lait et une sur 2 ou 3 un avortement.

L'amélioration des paramètres démographiques chez les juments apparaît donc fortement dépendante des problèmes de fertilité. Cela se complique chez le Pur Sang anglais par la nécessité d'amortir des reproductrices de prix élevé sur un faible nombre de produits. Il y a donc là un problème technique et un problème économique dont les résolutions conditionnent des progrès appréciables de la structure démographique femelle. En l'état actuel, seule une incitation à la mise à la reproduction précoce, soit jamais au-dessus de 5 ans, et à une réforme intensive à partir de Io ans, permettrait d'améliorer cette situation.

\section{CONCI,USION}

La structure démographique du Pur Sang en France apparaît dominée à l'issue de cette étude par de faibles performances de reproduction. Celles-ci, compensées par une grande durée d'utilisation des animaux, se traduisent globalement par un intervalle de génération long (10,5 ans) ce qui est le plus souvent considéré comme un handicap pour l'amélioration génétique. En revanche, dans leurs vies un étalon et une poulinière laissent respectivement de l'ordre de 50 et 5 descendants. Il y a donc, en tablant sur un sex-ratio de $r$ et ell se donnant une marge de 20 p. roo pour l'augmentation numérique de la population et la sélection naturelle, une possibilité de 
choix qu'on peut évaluer à $50 \mathrm{p}$. I00 chez les juments et à $5 \mathrm{p}$. I00 chez les étalons. Cela, si l'on considère que la grande majorité des Pur Sang sevrés sont soumis au contrôle individuel des performances, peut conduire à des intensités de sélection qui dépassent de loin ce qui est couramment appliqué chez les autres espèces domestiques. Toutefois, compte tenu de l'intervalle de génération, la population évolue lentement. En effet, le taux théorique de renouvellement annuel des poulinières n'est que d'environ 5 p. Ioo par an, celui des mâles étant donc de 0,5 p. Ioo. Sur une population qui est en France de l'ordre de 8 à ro ooo poulinières et de 6 à 700 étalons, cela correspond à un besoin théorique annuel de 4 à 500 juments et de 3 à 4 étalons. Ces chiffres qui sont, au moins pour les mâles, très inférieurs à la réalité laissent supposer que la sélection s'exerce non seulement par le contrôle individuel des performances mais également de façon non négligeable par le contrôle de la descendance. Sans doute l'excédent est-il rapidement éliminé pour caractéristiques de reproduction insuffisantes lorsqu'il s'agit de poulinières ou pour production insuffisante en quantité ou en qualité lorsqu'il s'agit d'étalons.

Cette structure démographique favorisant 1'intensité de sélection au détriment de l'intervalle de génération correspond-elle à un optimum du point de vue de la sélection? C'est probable chez les femelles où, sauf innovations importantes dans les techniques de reproduction, peu de progrès peuvent être espérés. En effet, seule une réforme beaucoup plus active des poulinières peu fertiles ou âgées permettrait, nous semble-t-il, de les obtenir. Chez les mâles en revanche beaucoup d'améliorations sont possibles. Le nombre annuel moyen de descendants par étalon est en effet très faible $(7,03)$ et leur mise à la reproduction tardive $(6,9$ ans). La réduction de l'effectif des étalons et la définition d'un statut spécial pour les étalons "de croisement " nous paraissent donc devoir s'imposer. En effet, c'est surtout sur l'intensité de sélection effectivement réalisée qu'un progrès génétique sensible peut être attendu dans cette population. La diminution de l'intervalle de génération ne peut dans les conditions actuelles être raisonnablement espérée car elle nécessiterait une forte réduction de la durée d'utilisation des reproducteurs, ce que les éleveurs ne sont pas prêts à accepter. Remarquons toutefois que l'insémination artificielle permettrait d'augmenter considérablement le nombre de descendants d'un étalon tout en réduisant au maximum sa durée d'utilisation. Ellle serait donc, nous semble-t-il, un apport très appréciable. Malheureusement, son emploi chez les chevaux de sang se heurte à des difficultés qu'il ne nous est pas possible d'analyser ici dans le détail, mais au premier rang desquelles figurent des considérations commerciales difficiles à modifier.

Reçu pour publication en septembre 1976.

\section{SUMMARY}

\section{ESTIMATION OF SOME DEMOGRAPHICAL PARAMETERS}

OF THOROUGHBRED HORSES IN FRANCE

The study based on the data of the French Stud-Book allowed to estimate to I0.5 years the generation interval in Thoroughbreds. This high value depends, on the one hand, on the mean age at first mating of the males $(6.9$ years $; n=23 \mathrm{I})$ and females $(4.9$ years $; n=930)$ and, on the other, on the late culling of these animals : 16.3 years on an average in the males $(n=477)$ and I4.3 years in the females $(n=930)$. 
Furthermore, a stallion produces on an average 7.03 weaned foals per year corresponding to an annual fertility rate of 0.58 for the mares. The mean number of foals produced by a stallion during its whole reproductive life is $\mathbf{5 2 . 4}$, that of a mare is 5.3 .

As about $15 \mathrm{p}$. 1oo of the males only start their reproductive life at an age of 9 years or more and as about $30 \mathrm{p}$. Ioo of the stallions supply only $2.5 \mathrm{p}$. Ioo of the offsprings, it should be possible by a better breeding management to substantially reduce the generation interval through the two paternal ways.

For the females the theoretical annual turnover rate ranges around $5 \mathrm{p}$. Ioo. Consequently it seems possible by culling more severely the least fertile or aged mares to act by this way on the generation interval.

The mean number of animals available for progeny-testing is small for stallions and negligeable for mares.

In conclusion, the organization of reproduction in the French population of thoroughbreds can be improved. Whithout modifying the selection methods this would allow to obtain a more rapid genetic progress.

\section{RÉFÉRENCES BIBLIOGRAPHIQUES}

Arthur G. H., r958. An analysis of the reproductive function of mares based on post mortem examina tion. Vet. Rec., 70, 682-686 (cité d'après PAlmer, 1972).

BAIN A. M., 1969. Foetal losses during pregnancy in the Thoroughbred mare : a record of 2562 pregnancies. N. Z. Vet. J., 17, I55-I 58 (cité d'après Palmer, 1972).

Belliti E., Pagano M. G., 1967. Indagine su alcuni parametri biologici del cavallo di Capitanata. Ann. Fac. Agric. Univ. Bari, 21, 185-206.

Carvahlo (de) R. L., Enne G., Coury A. Jr, 1972. Aspetti riproduttivi di equini di razza Mangalarga Paulista. Riv. Agric. Subtrop. Trop., 66, 99-1 Io.

Comfort A., 1958. The longevity and mortality of Thoroughbred mares. J. Gerontol., 13, 342-350.

Comfort A., 1959. The longevity and mortality of Thoroughbred stallions. J. Gerontol., 14, 9-Io.

Comfort A., 1962. A life table for Arabian mares. J. Gerontol., 17, 14.

Detrens S., Konopinski T., r954. Durée de vie reproductive des poulinières inscrites de la race de Poznan (polonais). Pr. Zakl. Szczegolowej Hodowli Zwierzat W.S.R. Wroclawiu Med. Wet., 10, 327-328. (A bstr. in Anim. Breed. Abstr., 22, n' 13 Io.)

Franciscis (de) G., Lella (di) T., Barbieri V., Pitaro E., r972. Indagine su alcuni parametri inerenti l'efficienza riproduttiva della popolazione equina $A$ velignese allevata nel mezzogiorno d'Italia. Acta Med. Vet., 18, I77-I96.

Koroljkov A. G., r951. Les jumeaux dans l'élevage du cheval (russe). Konevodstvo, (3), $20-22$ (Abstr. in A nim. Breed A bstr., 19, n' 1559).

Lauvergne J.-J., Boyazoglu J. G., Carta R., Casu S., 1973. Caractéristiques démographiques de la race ovine Sarde. Ann. Génét. Sél. anim., 5, 53-72.

Legault C., Dagorn J., 1973. Incidence de l'age à la première mise bas sur la productivité de la truie. Journ. rech. porcine, Fr., 227-237.

Manunta G., r953. Sulla durata della vita utile del cavallo. Arch. Vet. Ital., 4, 43-50.

Matassino D., I962. Studio sulle statistiche vitali nelle cavalle di razza Avelignese nel Mezzogiorno. 1. Inseminazioni, concepimenti, vuoti, aborti, parti ed età media ai diversi parti. Ann. Fac. Sci. A gric. Portici, Ser. 3, 28, 249-267. II. Intervallo interparto e sue frazioni, e loro ripetibilità ed eredibilità.

Ann. Fac. Sci. Agric. Portici, Ser. 3, 28, 269-285.

Nishikawa Y., Hafez E. S. E., I962. The Reproduction of Horses, in Hafez, Reproduction of Farm Animals. Lea and Febiger, Philadelphia, chap. 16, 266-276.

Palmer E., 1972. Etude bibliographique de la reproduction chez la Jument. Stn. Physiol. Reprod., Nouzilly, I.N.R.A., France, ronéoté p. 35.

Salerno A., Montemurro N., r965. Ricerche su alcune statistiche vitali nella razza equina Salernitana. Ripetibilità dell'età al primo parto. Atti. Soc. Ital. Sci. Vet., 18, 294-296.

SAsımowski E., r961. Durée d'utilisation à l'élevage des étalons de sang pur dans le district de Lublin (polonais). Ann. Univ. M.-Curie-Sklodowska, E, 14, $321-337$ (A bstr. in Anim. Breed. Abstr., 81, $\mathrm{n}^{\circ}$ 43).

SteEle D. G., 1944. A genetic analysis of recent thoroughbreds, Standardbreds, and American saddle horses. Ky. A gric. Exp. Stn., Bull., 462, 27.

Wiltbank J. N., Gregory K. E., Swiger L. A., Ingalls J. E., Rothlisberger J. A., Koch R. M., r966. Effects of heterosis on age and weight at puberty in beef Heifers. J. anim. Sci., 25, 744-75r.

Wussow W., Hartwig W., r956. Der Einfluss des Alters auf die Fruchtbarkeit der Stuten. Tierzucht, 10, 344-345. 
Zwolinski J., 1966. Analyse de quelques phenomenes lies a la reproduction chez les juments (polonais).

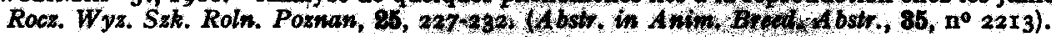

Zwonisski J., DrzewieckI W., 1966. Durce de vie et utilisation des etalons d'élite dans la province de Poznan (polonais). Rocz. wyz. Szk. roln. Poznan, 85, 233-240. (Abstr. in Anim. Breed A bstr., 86, n 2214.) 